\title{
Knowledge, attitude, and practices towards schistosomiasis among rural population in Yemen
}

Hany Sady ${ }^{1,2}$, Hesham M. Al-Mekhlafi ${ }^{1,3,4^{*}}$, Wahib M. Atroosh ${ }^{1}$, Ahmed K. Al-Delaimy ${ }^{1}$, Nabil A. Nasr ${ }^{1}$, Salwa Dawaki ${ }^{1}$, Mona A. Al-Areeqi ${ }^{1}$, Init Ithoi ${ }^{1}$, Awatif M. Abdulsalam ${ }^{1}$, Kek Heng Chua ${ }^{5}$ and Johari Surin ${ }^{1}$

\begin{abstract}
Background: Schistosomiasis is highly prevalent in Yemen, with an estimated 3 million cases, particularly among rural communities. This community-based study aims to evaluate the knowledge, attitude and practices (KAP) on schistosomiasis among rural communities in Yemen.

Methods: A cross-sectional study was carried out among 250 households from ten rural districts in Yemen. Overall, 400 children were screened for urogenital and intestinal schistosomiasis. Moreover, parents were interviewed using a pre-tested questionnaire to collect information about the demographic and socioeconomic information and their KAP concerning schistosomiasis.

Results: A total of 127 (31.8\%) children were found to be excreting schistosome eggs in either their urine or faeces ( $22.5 \%$ S. haematobium and $8.0 \%$ S. mansoni). Although $92.4 \%$ of the respondents had heard about schistosomiasis, $49.8 \%, 68.0 \%$ and $47.2 \%$ had knowledge concerning the transmission, signs and symptoms, and prevention, respectively. In addition, $77.1 \%$ considered schistosomiasis as harmful while $48.5 \%$ believed that schistosomiasis could be prevented, albeit their practices to prevent infections were still inadequate. Significant associations between the KAP and age, education, employment status and household monthly income were reported $(P<0.05)$. Moreover, a significantly higher level of knowledge was reported among the respondents who had infected children compared to those with no infected family members $(P<0.05)$. Multiple logistic regression analysis revealed that the level of education and the history of schistosomiasis were the most important factors associated with the KAP concerning schistosomiasis among this population.

Conclusion: This study reveals that knowledge about the cause, transmission, symptoms and prevention of schistosomiasis among the rural population in Yemen was inadequate, and that this could be a challenging obstacle to the elimination of schistosomiasis in these communities. Besides the current mass drug administration, school and community-based health education regarding schistosomiasis is imperative among these communities to significantly reduce the transmission and morbidity of schistosomiasis.
\end{abstract}

Keywords: Knowledge, Attitude, Practice, Schistosomiasis, Neglected tropical diseases, Yemen

\footnotetext{
*Correspondence: halmekhlafi@yahoo.com

'Department of Parasitology, Faculty of Medicine, University of Malaya, 50603

Kuala Lumpur, Malaysia

${ }^{3}$ Azal National Research Center, Azal University for Human Development, 447

Sana'a, Yemen

Full list of author information is available at the end of the article
}

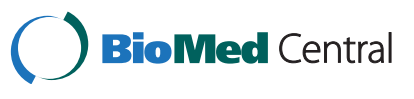

(c) 2015 Sady et al. Open Access This article is distributed under the terms of the Creative Commons Attribution 4.0 International License (http://creativecommons.org/licenses/by/4.0/), which permits unrestricted use, distribution, and reproduction in any medium, provided you give appropriate credit to the original author(s) and the source, provide a link to the Creative Commons license, and indicate if changes were made. The Creative Commons Public Domain Dedication waiver (http://creativecommons.org/publicdomain/zero/1.0/) applies to the data made available in this article, unless otherwise stated. 


\section{Background}

Schistosomiasis is one of the most serious and prevalent neglected tropical diseases (NTDs) in many developing countries, particularly in Africa (which has about $90 \%$ of the world's reported cases), Latin America and the Middle East. Recent estimates revealed that more than 200 million people are infected with Schistosoma species worldwide, and that almost 700 million people are at risk of this infection $[1,2]$. The disease is caused by different schistosoma species, with S. haematobium (causes urinary schistosomiasis), S. mansoni and S. japonicum (causes intestinal schistosomiasis) being the main and most common species. S. haematobium infection is characterized by haematuria as the classical sign. In chronic cases, bladder and ureteral fibrosis, sandy patches in the bladder mucosa and hydronephrosis occur while in advanced cases the infection is associated with bladder cancer [3-5]. Meanwhile, S. mansoni infection in humans causes diarrhoea, abdominal pain and blood in faeces. In the late stage, hepatosplenomegaly is the common complication with ascites and portal hypertension.

In December 2009, Yemen launched a six-year project to eliminate schistosomiasis-related morbidity and control intestinal worms throughout Yemen with financial support from the World Bank and WHO [6]. Referring to the funding agreement signed by the Ministry of Public Health and Population, Yemen, mass drug administration (MDA) was considered as the only intervention against schistosomiasis in Yemen [7]. However, health education materials (leaflets and posters) providing information on the transmission cycle and prevention of schistosomiasis and other helminth infections were also distributed during MAD campaigns [8]. The programme was disrupted by civil and political unrest resulting from "the Arab Spring" during 2010-2011 and re-commenced in 2012. Nonetheless, the current widespread civil war, which started in March 2015, has created more obstacles for the sustainability of the programme.

Despite the intensive control efforts by the government and international bodies, schistosomiasis is still a life-threatening public health problem in Yemen, with an estimated 3 million cases, which places the country second, after Egypt with 7 million cases, as having the highest prevalence of schistosomiasis in the Middle East $[2,9,10]$. Moreover, a recent study from Yemen showed that $59 \%$ of the squamous cell carcinoma (SCC) cases were caused by $S$. haematobium chronic infection among adults [11]. However, data on the knowledge, attitude, and practices (KAP) of populations in endemic areas in Yemen are not available. Community awareness and involvement are considered as one of the cardinal tools for the success and sustainability of any disease control programme [12]. In low socioeconomic communities, intervention through public awareness is often recommended as a first line of action to create the enabling environment for other strategies to thrive [13]. Moreover, such data are of great importance for identifying, designing and implementing effective community-based control interventions [14-16]. Within this context, the present study aims to evaluate the KAP towards schistosomiasis in Yemen. It is hoped that our findings will provide new information about the schistosomiasis-related KAP of the targeted population, and will add new insights about the prevention and control of this devastating disease in Yemen.

\section{Methods}

\section{Ethical consideration}

The present study was carried out according to the guidelines laid down in the Declaration of Helsinki and all procedures involving human subjects were approved by the Medical Ethics Committee of the University of Malaya Medical Centre, Malaysia (reference number: 968.4). The protocol was also approved by the University of Hodeidah, and permission was also given by the Yemen Schistosomiasis National Control Project and health offices of the relevant provinces. Before commencement of the study, information about the objectives of the study and the role of the participants were given to the invited people. Moreover, they were informed that their participation was voluntary and that they could withdraw from the study at any stage without any consequences. Subsequently, written and signed or thumb-printed informed consent was obtained from the heads of the households to conduct the study and also on behalf of their children; these procedures were approved by the Medical Ethics Committee of the University of Malaya. All the infected children were treated with a single dose of $40 \mathrm{mg} / \mathrm{kg}$ body weight praziquantel tablet.

\section{Study design and study area}

Being one of the poorest countries in the Middle East, Yemen has the highest percentage of people living in poverty with more than $50 \%$ of the population of nearly 25 million people being estimated to be under the national poverty line [17]. The country has been unstable for several years, suffering from civil wars, a deteriorating economy and severe depletion in water resources, with only $25 \%$ of the population having easy access to safe water.

A cross-sectional community-based study was conducted among the rural population in five different provinces in Yemen, namely Taiz, Ibb, Dhamar, Sana'a and Hodiedah. A questionnaire survey and sample collection was carried out between January and July 2012. In each province, two rural districts were randomly selected from the available official district list and then two villages within the selected districts were considered in collaboration with the Schistosomiasis Control Project office in each province (Fig. 1). The inclusion criteria in selecting these study areas were 


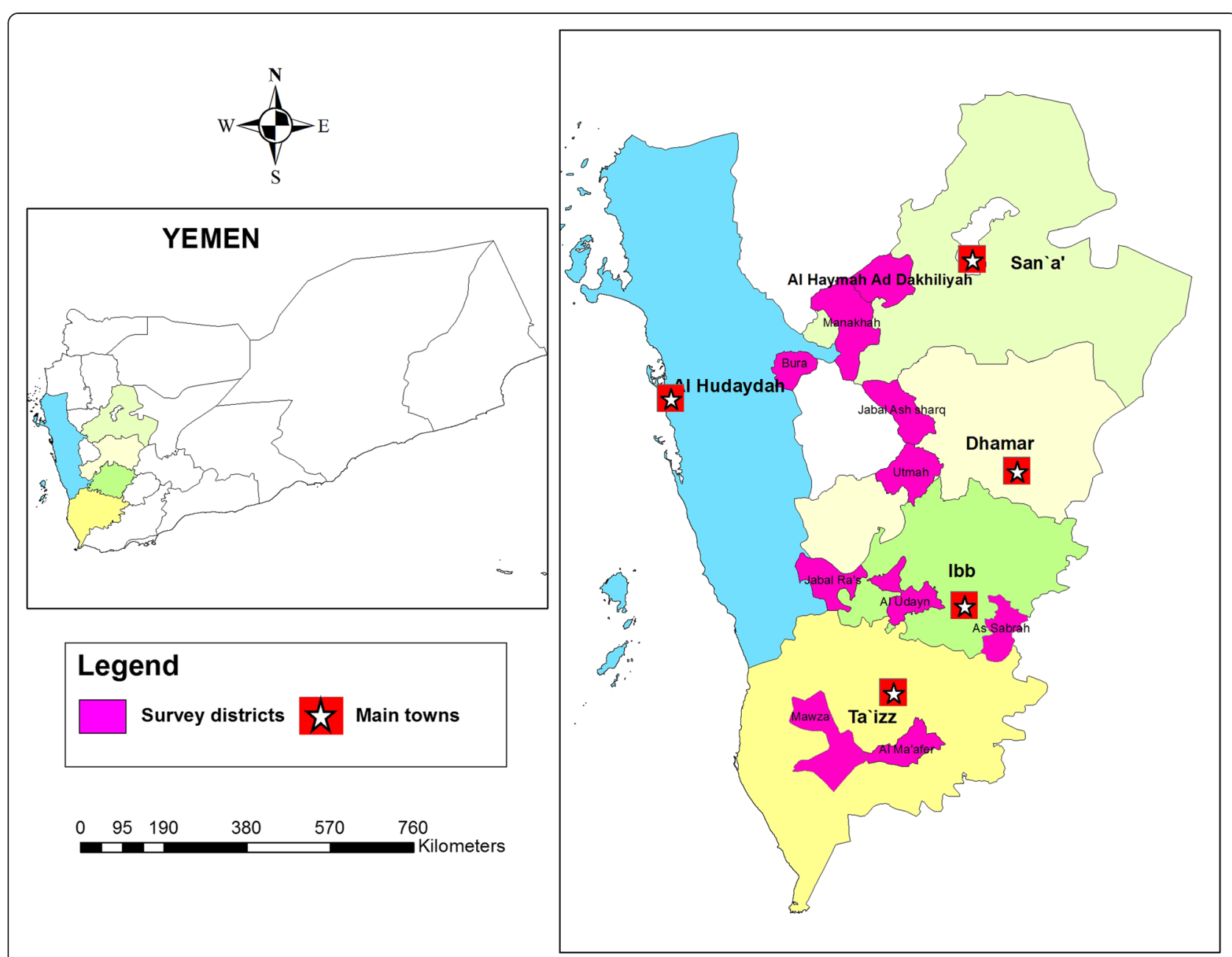

Fig. 1 A geographic map showing the study area in Yemen (ten districts within five provinces)

rural areas, > 50 households, undergoing active control surveillance and people's willingness to participate in the study. The districts were Mosa and Almafer (Taiz), Alsabrah and Alodien (Ibb), Otmah and Gabal al sharq (Dhamar), Alhemah and Manakhah (Sana'a), and Gabal Ras and Bora (Hodiedah). These areas were known to be endemic for schistosomiasis and undergoing active surveillances by the schistosomiasis national control project. The number of inhabitants per household was recorded and all were invited to participate in this study. Unique reference codes were assigned to each household and study participant.

\section{Study population}

Out of 780 households in the study areas, 250 households were randomly selected for this study ( 25 households from each village). At the village setting, a simple map was drawn for the distribution and location of houses and then every second house was selected for data collection. Of 632 children who were available in the selected houses and agreed to participate, a total of 430 (68.0 \%) children aged $\leq 15$ years delivered the containers for examination. Birth dates were obtained from the children's birth certificates or immunization records (available for most of those aged below 10 years) or from any related official document available from the parents. In this study, 202 (32.0\%) failed to submit samples and/or were absent during the questionnaire survey, and 30 (4.7\%) containers were returned empty. Hence, they were excluded from the study. Overall, 400 $(63.3 \%)$ children (59.5\% males and $40.5 \%$ females) who delivered suitable samples for examination together with complete questionnaire data were included in this study.

\section{Questionnaire survey}

A validated questionnaire was used to collect data about the demographic, socio-economic, environmental background, personal hygiene, water contact and collection practices, and history of receiving anti-schistosomal treatment. Moreover, the questionnaire also involved questions concerning the knowledge about schistosomiasis aetiology, 
transmission, clinical manifestations, prevention and control. Trained research assistants collected information from the children's parents via face-to-face interviews. The survey gathered information on both spontaneous and probed knowledge about schistosomiasis. Questions on knowledge were open-ended questions, without multiple-choice answers to avoid guessing, which might have given a false impression concerning the knowledge of the participant. However, the questions pertaining to the practices were provided with multiple-choice answers to assess the frequency of performing these activities or actions. At the end of each interview session, the interviewer probed for further knowledge related to schistosomiasis and not mentioned spontaneously by the respondent. However, this was made on selected variables only, specifically the source of information about the disease, history and reasons for previous visits to clinics or hospitals, reasons for not swimming in open water, and the identification of snails and whether or not they are harmful upon showing them to the respondents. The questionnaire was prepared in English and then translated into Arabic (the native language of Yemen). During the survey, direct observations were made concerning the cleanliness of the houses (such as availability of functioning toilets, washing soap, piped water, water containers and cisterns), and the personal hygiene of children (such as nail cutting, clothes, clean hands, wearing shoes when going outside the house).

\section{Parasitology}

Faecal and urine samples were collected from children in $100 \mathrm{ml}$ clean, labelled, and screw-capped containers. The samples were collected between 9-11 a.m. and transported within $5 \mathrm{~h}$ to the nearest laboratory in suitable cool boxes $\left(4-6{ }^{\circ} \mathrm{C}\right)$. The faecal samples were examined using formalin ether sedimentation and Kato-Katz techniques for the presence of S. mansoni eggs [18]. Meanwhile, the urine samples were examined using the dipstick test and sedimentation method using a Nuclepore membrane for the presence of S. haematobium eggs [19]. To determine the worm burden, egg counts were taken and recorded as eggs per gram of faeces (EPG) for each positive sample and the intensity of infections was graded as heavy ( $\geq 400 \mathrm{EPG}$ ), moderate (100-399 EPG) or light (1-99 EPG) according to the criteria proposed by the WHO [20]. Similarly, egg counts were taken and recorded as eggs/10 $\mu \mathrm{l}$ urine (EP10ml), and the intensity of the infection was graded as heavy ( $>50 \mathrm{EP} 10 \mathrm{ml})$ or light $(1-50 \mathrm{EP} 10 \mathrm{ml})$ [20]. For quality control, $25 \%$ of the samples were selected randomly and re-examined by another assistant to confirm the results.

\section{Data analysis}

Data were double entered by two different researchers into Microsoft Office Excel 2007 spreadsheets. Then, a third researcher crosschecked the two datasets for accuracy and created a single dataset. Data analysis was performed using IBM SPSS Statistics, version 18.0 (IBM Corporation, NY, USA). For descriptive analysis, the percentage was used to express the prevalence of infections and proportion of variable categories. Although the egg counts were found to be not normally distributed, there are biological justifications for using the arithmetic mean ( \pm standard deviation, SD) rather than the median or geometric mean to express the egg count of each Schistosoma species [21]. Pearson's Chi Square $\left(\chi^{2}\right)$ test or Fisher's exact test was used where appropriate to examine the difference in proportions between groups and to test the association between KAP and the demographic, socioeconomic and infection status. Odds ratios (ORs) and $95 \%$ confidence intervals (CIs) were computed for all variables and $P<0.05$ was considered as the level of significance. Multiple logistic regression analysis was performed to identify the factors significantly associated with the KAP variables among the studied population. A $P$ value of $<0.05$ was considered to be statistically significant.

\section{Results}

In total, 250 Yemeni householders with a mean age of $42 \pm 4.3$ years were interviewed face-to-face to fill in the questionnaire on their KAP towards schistosomiasis (Table 1). Due to cultural customs, the majority (78.1\%) of the respondents were males, and more than half of the fathers had at least primary education (6 years of formal education). About two-thirds of the participants had low family monthly income $(<$ YER20, 000; US\$1 = YER

Table 1 Demographic and socioeconomic characteristics of the subjects who participated in the study $(n=250)$

\begin{tabular}{ll}
\hline Characteristics & $\mathrm{n}(\%)$ \\
\hline Age groups & \\
$\quad<40$ years & $114(45.4)$ \\
$\geq 40$ years & $136(54.6)$ \\
Gender & \\
Males & $195(78.1)$ \\
Females & $55(21.9)$ \\
Socioeconomic status & \\
Educated fathers (at least primary) & $141(56.3)$ \\
Low household income (<YER20, 000) & $157(62.9)$ \\
Farmers and non working fathers & $142(57.0)$ \\
Large family size (>7 members) & $124(49.5)$ \\
Piped water supply & $77(30.9)$ \\
Electricity & $72(28.9)$ \\
Presence of toilet in house & $137(54.9)$ \\
History of schistosomiasis & $126(50.5)$ \\
\hline
\end{tabular}

All values are number (\%). YER, Yemen Riyal; (US\$1 = YER216) 
216). Less than one-third (30.9 \%) of the houses had a piped water supply while about one quarter of the houses had electricity (mainly during night-time).

\section{Prevalence and distribution of schistosomiasis}

Of the 400 participants, 127 (31.8\%) were egg-positive for schistosomiasis. Overall, 90 participants $(22.5 \%)$ had urogenital schistosomiasis, $32(8.0 \%)$ had intestinal schistosomiasis and $5(1.3 \%)$ were co-infected with both S. haematobium and S. mansoni. Of the 95 S. haematobium-positive samples, 21 (21.1\%) were of heavy intensity with a mean EP10ml of $340( \pm 244)$ eggs while 74 $(77.9 \%)$ cases were of light intensity with a mean EP10ml of $17( \pm 10)$ eggs. Likewise, $15(40.5 \%)$ and 3 (8.1\%) S. mansoni cases were of moderate and heavy intensity with a mean of EPG of $212( \pm 82)$ and $637( \pm 93)$ eggs, respectively. Moreover, 19 (51.4 \%) S. mansoni cases were light infections with a mean EPG of $50( \pm 23)$ eggs. Data on the prevalence, distribution and key factors significantly associated with schistosomiasis have been published previously in Sady et al. [10].

\section{Knowledge about schistosomiasis, its signs and symptoms, transmission and prevention}

The results about the knowledge of the participants regarding schistosomiasis transmission, signs and symptoms and prevention are shown in Table 2. It was found that a majority of 231 (92.4\%) of the respondents had heard about schistosomiasis (locally known as Bilharsia). Moreover, $68.0 \%(157 / 231)$ mentioned at least one sign or symptom related to the disease, of whom 92 (39.8\%) mentioned haematuria, 90 (39.0\%) mentioned abdominal pain, and 41 (17.7\%) mentioned blood in stools; $32.0 \%$ could not cite any symptom.

The results further showed that there was a lack of knowledge about the transmission of schistosomiasis among these people in that only $49.8 \%(115 / 231)$ of the respondents were able to mention at least one mode of transmission. Of these, $28.6 \%$ correlated the disease with contact with infested water while $11.3 \%(26 / 231)$ indicated the role of snail vectors. Concerning the knowledge about the prevention of schistosomiasis, $47.2 \%$ $(109 / 231)$ were able to give at least one measure of prevention, with $26.8 \%$ mentioning avoiding swimming in ponds/ streams, $10.4 \%$ avoiding washing clothes in ponds/streams, and $18.6 \%$ mentioned taking anti-schistosomal drugs.

\section{Attitude and practices of people in rural Yemen to schistosomiasis}

Table 3 shows the results about the attitude and practices of the respondents towards schistosomiasis. Of the participants who had prior knowledge concerning schistosomiasis, $77.1 \%(178 / 231)$ considered schistosomiasis as a serious disease. Surprisingly, less than half (48.5\%)
Table 2 Knowledge about schistosomiasis, symptoms, transmission and prevention among rural population in Yemen who had prior knowledge on schistosomiasis $(n=231)$

\begin{tabular}{|c|c|c|}
\hline Variable & Number & Percent \\
\hline \multicolumn{3}{|l|}{ Source of information } \\
\hline Health centre for schistosomiasis control & 154 & 66.8 \\
\hline Health clinic/hospitals & 50 & 21.7 \\
\hline Mass media & 5 & 2.2 \\
\hline School & 4 & 1.7 \\
\hline Do not remember & 18 & 7.6 \\
\hline \multicolumn{3}{|l|}{ Signs and symptoms } \\
\hline Haematuria & 92 & 39.8 \\
\hline Abdominal pain & 90 & 39.0 \\
\hline Diarrhoea & 83 & 35.9 \\
\hline Fever & 64 & 27.7 \\
\hline Burning urination & 42 & 18.2 \\
\hline Blood in stool & 41 & 17.7 \\
\hline Vomiting & 35 & 15.2 \\
\hline Itching & 34 & 14.7 \\
\hline Pale face (anaemia) & 33 & 14.3 \\
\hline Fatigue & 33 & 14.3 \\
\hline Loss of appetite & 31 & 13.4 \\
\hline Cough & 28 & 12.1 \\
\hline Swollen abdomen & 25 & 10.8 \\
\hline Dysentery & 17 & 7.4 \\
\hline Do not know & 74 & 32.0 \\
\hline \multicolumn{3}{|l|}{ Transmission } \\
\hline Playing with soil & 76 & 32.9 \\
\hline Swimming/bathing in infested water & 66 & 28.6 \\
\hline Dirty hands & 56 & 24.2 \\
\hline Eating contaminated food & 55 & 23.8 \\
\hline Drinking untreated water & 34 & 14.7 \\
\hline Snail & 26 & 11.3 \\
\hline Do not know & 116 & 50.2 \\
\hline \multicolumn{3}{|l|}{ Prevention } \\
\hline Avoid playing with soil & 72 & 31.2 \\
\hline Washing hands before eating & 65 & 28.1 \\
\hline Avoid swimming/bathing in ponds/streams & 62 & 26.8 \\
\hline Washing vegetables/fruit before eating & 53 & 22.9 \\
\hline Taking anti-schistosomal drugs & 43 & 18.6 \\
\hline Avoid drinking untreated water & 33 & 14.3 \\
\hline Avoid washing clothes in ponds/streams & 24 & 10.4 \\
\hline Do not know & 122 & 52.8 \\
\hline
\end{tabular}

of the respondents believed that schistosomiasis can be prevented and only $13.0 \%$ agreed about the role of faeces and urine as the source of infection. With regards to 
Table 3 Attitude and practices of the participants towards schistosomiasis in rural Yemen

\begin{tabular}{|c|c|c|}
\hline Variables & Number & Percent \\
\hline \multicolumn{3}{|l|}{ Is schistosomiasis a serious disease? $(n=231)$} \\
\hline Yes & 178 & 77.1 \\
\hline No & 15 & 6.5 \\
\hline Do not know & 38 & 16.4 \\
\hline \multicolumn{3}{|l|}{$\begin{array}{l}\text { Believes that schistosomiasis can be prevented } \\
(n=231)\end{array}$} \\
\hline Yes & 112 & 48.5 \\
\hline No & 87 & 37.7 \\
\hline Do not know & 32 & 13.9 \\
\hline \multicolumn{3}{|l|}{ Faeces/urine as a source of infections $(n=231)$} \\
\hline Yes & 30 & 13.0 \\
\hline No & 26 & 11.2 \\
\hline Do not know & 175 & 75.8 \\
\hline \multicolumn{3}{|l|}{ Practices $(n=250)$} \\
\hline Swimming/bathing in open water source & 147 & 58.8 \\
\hline Indiscriminate defaecation/urination & 148 & 59.2 \\
\hline Fetching water from ponds/streams & 139 & 55.5 \\
\hline Washing clothes or utensil in open water source & 83 & 33.3 \\
\hline Wearing shoes when going outside & 186 & 74.3 \\
\hline Drinking untreated water & 173 & 69.1 \\
\hline $\begin{array}{l}\text { Seeking treatment from clinic for GIT and urinary } \\
\text { tract symptoms }\end{array}$ & 203 & 81.1 \\
\hline $\begin{array}{l}\text { Seeking traditional treatment for GIT and urinary } \\
\text { tract symptoms }\end{array}$ & 36 & 14.3 \\
\hline Do nothing for GIT and urinary tract symptoms & 11 & 4.5 \\
\hline
\end{tabular}

the practices of these people, the results showed that $58.8 \%(147 / 250)$ of the respondents swim or bathe in open water and $59.2 \%(148 / 250)$ practice open defaecation and/or urination in the water and fields. Likewise, 139 (55.5\%) of the respondents fetch water from ponds/ streams/pumps/ wells and 83 (33.3\%) wash clothes in such water. In terms of treatment-seeking behaviour, $81.1 \%(203 / 250)$ of the respondents mentioned that they go to the nearest clinic/hospital for treatment in case of gastrointestinal symptoms or haematuria while $4.5 \%$ do nothing. Interestingly, $14.3 \%(36 / 250)$ of the participants revealed that they go to traditional healers for the treatment of such signs and symptoms; however, none were able to mention the name of any medicinal plant or traditional remedy they had used.

\section{Association of knowledge of the participants about schistosomiasis with some demographic and socioeconomic factors}

Table 4 shows that a significantly lower percentage of those aged below 40 years had heard about schistosomiasis compared to those aged $\geq 40$ years ( $88.6 \%$ vs. $95.6 \%$;
$P=0.038)$. However, knowledge of fatigue $(P=0.021)$, swollen abdomen $(P=0.031)$ and vomiting $(P=0.001)$ as symptoms of schistosomiasis was significantly higher in those aged below 40 years compared to those aged $\geq$ 40 years. Similarly, the percentage of educated parents who had heard about schistosomiasis was significantly higher than that for non-educated parents $(96.5 \%$ vs. $87.2 \% ; P=0.006)$. It was found that $73.8 \%$ of the educated respondents had knowledge about at least one symptom of the disease compared to $49.1 \%$ of their non-educated counterparts $(P<0.001)$. Likewise, respondents who had at least 6 years of formal education had a significantly higher level of knowledge about the role of snails as a mode of transmission of schistosomiasis $(P=0.036)$, and about avoiding swimming/playing in open water $(P=0.003)$, as well as about taking anti-schistosomal drugs as preventive measures $(P=0.001)$ compared to the non-educated respondents.

Table 5 shows that the percentage of respondents who had heard about schistosomiasis was significantly higher among the working respondents $(97.2 \%$ vs. $88.7 \%$; $P=$ 0.012 ) and those who had a household monthly income of $\geq$ YR20, 000 (97.8 \% vs. $89.2 \%$; $P=0.012$ ) when compared to the non-working residents and those who had low household monthly income. Likewise, the results revealed a significantly higher level of knowledge about the signs and symptoms among the working respondents and those with a household monthly income of $\geq$ YR20, 000 compared to their counterparts.

\section{Association of attitude and practices of the participants towards schistosomiasis with some demographic and socioeconomic factors}

Tables 6 and 7 show a significant association of attitude towards schistosomiasis with the age of the respondents $(P=0.030)$, while the association of attitude with the educational level, employment status and household monthly income of the respondents was not significant $(P>0.05)$. Moreover, practising indiscriminate defaecation/urination ( $66.7 \%$ vs. $52.9 \% ; P=0.028$ ) and clothes or utensil washing in open water $(41.6 \%$ vs. $26.3 \%$; $P=0.010)$ were found to be significantly higher in the respondents aged below 40 years than those aged $\geq 40$ years (Table 6). On the other hand, practising indiscriminate defaecation/urination was significantly lower among the respondents who were educated (44.3\% vs. $78.2 \% ; P<0.001)$, working (37.4 \% vs. $75.5 \% ; P<0.001)$ and with a household monthly income of $\geq$ YR20, 000 (30.1 \% vs. 76.4 \%; $P<0.001)$ compared to their counterparts.

\section{Association of KAP of the participants on schistosomiasis} with the infection status among their children

The association between the knowledge of participants on schistosomiasis (prior knowledge concerning 
Table 4 Associations of knowledge of the participants about schistosomiasis with their age and educational level

\begin{tabular}{|c|c|c|c|c|c|c|c|c|}
\hline \multirow[t]{2}{*}{ Variable } & \multicolumn{4}{|c|}{ Age (years) } & \multicolumn{4}{|c|}{ Educational level } \\
\hline & $>=40$ & $<40$ & OR & $95 \% \mathrm{Cl}$ & Non educated & Educated & OR & $95 \% \mathrm{Cl}$ \\
\hline Heard about schistosomiasis & 130(95.6) & $101(88.6)$ & 0.4 & $0.1,0.9^{*}$ & $95(87.2)$ & 136(96.5) & 4.0 & $1.4,11.5^{*}$ \\
\hline \multicolumn{9}{|l|}{ Signs and symptoms: } \\
\hline At least one symptom & $88(64.7)$ & $69(61.1)$ & 0.9 & $0.5,1.4$ & $53(49.1)$ & 104(73.8) & 2.9 & $1.7,4.9^{*}$ \\
\hline Haematuria & $47(34.6)$ & 45(39.8) & 1.3 & $0.7,2.1$ & $30(27.5)$ & $62(44.0)$ & 2.1 & $1.2,3.5^{*}$ \\
\hline Abdominal pain & $52(38.2)$ & $37(32.7)$ & 0.8 & $0.5,1.3$ & $35(32.1)$ & 55(39.0) & 1.4 & $0.8,2.3$ \\
\hline Burning urination & $22(16.2)$ & $20(17.5)$ & 1.1 & $0.6,2.2$ & $16(14.7)$ & 26918.4) & 1.3 & $0.7,2.6$ \\
\hline Blood in stool & 26(19.0) & $16(14.0)$ & 0.7 & $0.4,1.4$ & $11(10.1)$ & $30(21.4)$ & 2.4 & $1.2,5.1^{*}$ \\
\hline Itching & $17(12.4)$ & $17(14.9)$ & 1.2 & $0.6,2.6$ & $12(11.0)$ & $22(15.6)$ & 1.5 & $0.7,3.2$ \\
\hline Pale face (anaemia) & 15(10.9) & 19(16.7) & 1.6 & $0.8,3.4$ & 13(11.9) & $20(14.3)$ & 1.2 & $0.6,2.6$ \\
\hline Fatigue & $11(8.0)$ & $20(17.7)$ & 2.5 & $1.1,5.4^{*}$ & 14(12.8) & $17(12.1)$ & 0.9 & $0.4,2.0$ \\
\hline Swollen abdomen & $9(6.6)$ & $17(14.9)$ & 2.5 & $1.1,5.8^{*}$ & $10(9.2)$ & 15(10.6) & 1.2 & $0.5,2.7$ \\
\hline Diarrhoea & $44(32.1)$ & $39(34.5)$ & 1.1 & $0.7,1.9$ & $25(22.7)$ & $58(41.4)$ & 2.4 & $1.4,4.2^{*}$ \\
\hline Loss of appetite & 19(13.9) & $14(12.4)$ & 0.9 & $0.4,1.8$ & $11(10.0)$ & $22(15.6)$ & 1.7 & $0.8,3.6$ \\
\hline Fever & $35(25.5)$ & $29(25.7)$ & 1.0 & $0.6,1.8$ & 21(19.3) & $43(30.5)$ & 1.8 & $1.0,3.3^{*}$ \\
\hline Vomiting & $8(5.8)$ & 23(20.4) & 4.1 & $1.8,9.6^{*}$ & $11(10.0)$ & 21(14.9) & 1.6 & $0.7,3.4$ \\
\hline Dysentery & $11(8.0)$ & $6(5.3)$ & 0.6 & $0.2,1.8$ & $3(2.8)$ & $13(9.2)$ & 3.6 & $1.0,12.9^{*}$ \\
\hline \multicolumn{9}{|l|}{ Transmission: } \\
\hline At least one way of transmission & $56(45.9)$ & $59(45.7)$ & 1.0 & $0.6,1.6$ & $45(41.3)$ & $69(48.9)$ & 1.4 & $0.8,2.3$ \\
\hline Swimming/bathing/playing in infested water & $35(28.7)$ & $31(24.2)$ & 0.8 & $0.5,1.4$ & $24(22.0)$ & $42(29.8)$ & 1.4 & $0.8,2.7$ \\
\hline Snail & $11(9.1)$ & 14(10.9) & 1.2 & $0.5,2.8$ & $6(5.5)$ & 19(13.6) & 2.7 & $1.1,7.0^{*}$ \\
\hline Playing with soil & $40(32.8)$ & $37(28.9)$ & 0.8 & $0.5,1.4$ & 29(26.6) & $48(34.0)$ & 1.4 & $0.8,2.5$ \\
\hline Dirty hands & $27(22.3)$ & $29(22.7)$ & 1.0 & $0.6,1.9$ & 19(17.4) & $38(27.0)$ & 1.7 & $0.9,3.2$ \\
\hline Eating contaminated food & $25(20.5)$ & $31(24.2)$ & 1.2 & $0.7,2.3$ & 19(17.3) & $38(27.0)$ & 1.8 & $0.9,3.3$ \\
\hline Drinking untreated water & 16(13.1) & 18(14.1) & 1.1 & $0.5,2.2$ & $7(6.4)$ & 26(18.6) & 3.3 & $1.4,8.0^{*}$ \\
\hline \multicolumn{9}{|l|}{ Prevention: } \\
\hline At least one preventive measure & $55(45.1)$ & $58(45.3)$ & 1.0 & $0.6,1.7$ & $35(31.8)$ & $79(56.0)$ & 2.7 & $1.6,4.6^{*}$ \\
\hline Avoid swimming/playing in ponds/streams & $38(31.1)$ & $27(21.1)$ & 0.6 & $0.3,1.0$ & $18(16.5)$ & 47(33.3) & 2.5 & $1.4,4.7^{*}$ \\
\hline Avoid washing clothes in ponds/streams & $12(9.8)$ & 14(10.9) & 1.2 & $0.5,2.5$ & 11(10.1) & 15(10.6) & 1.1 & $0.5,2.4$ \\
\hline Avoid playing with soil & $39(32.0)$ & $33(25.8)$ & 0.7 & $0.4,1.3$ & $25(22.7)$ & $47(33.3)$ & 1.7 & $0.9,3.0$ \\
\hline Washing hands before eating & $31(25.6)$ & $35(27.3)$ & 1.1 & $0.6,1.9$ & $22(20.0)$ & 45(31.9) & 1.9 & $1.1,3.4^{*}$ \\
\hline Avoid drinking untreated water & 14(11.5) & 19(14.8) & 1.3 & $0.6,2.8$ & 10(9.1) & 23(16.4) & 2.0 & $0.9,4.3$ \\
\hline Washing vegetables/fruit before eating & 29(23.8) & 24(18.8) & 0.7 & $0.4,1.4$ & 18(16.5) & $35(24.8)$ & 1.7 & $0.9,3.1$ \\
\hline Taking anti-schistosomal drugs & 21(17.4) & $21(16.4)$ & 0.9 & $0.5,1.8$ & $9(8.3)$ & $33(23.6)$ & 3.4 & $1.6,7.5^{*}$ \\
\hline
\end{tabular}

All values are number (\%). OR, Odds ratio. $\mathrm{Cl}$, Confidence interval. *Significant association $(P<0.05)$

the signs and symptoms, transmission and prevention) and the infection status among their children was investigated. The results showed that the knowledge about haematuria $(P=0.042)$, burning urination $(P=0.045)$ and pale face (anaemia) $(P=0.017)$ as signs and symptoms of schistosomiasis, and avoiding washing clothes in open water sources as a preventive measure $(P=0.12)$ was significantly higher among those who had infected children compared to those with no infected family members. Likewise, a higher level of knowledge about blood in stools as a symptom $(23.4 \%$ vs. $15.6 \%)$, and swimming or bathing in open water sources (13.0 \% vs. $6.5 \%)$ and snails (15.8 \% vs. $8.4 \%)$ as modes of transmission of schistosomiasis was reported among the respondents who had infected children compared to those with non-infected children; however, the difference was not statistically significant $(P>0.05)$. 
Table 5 Associations of knowledge of the participants about schistosomiasis with their employment status and household monthly income

\begin{tabular}{|c|c|c|c|c|c|c|c|c|}
\hline \multirow[t]{2}{*}{ Variable } & \multicolumn{4}{|c|}{ Employment status } & \multicolumn{4}{|c|}{ Household monthly income } \\
\hline & Not working & Working & OR & $95 \% \mathrm{Cl}$ & $<Y R 20,000$ & $\geq Y R 20,000$ & OR & $95 \% \mathrm{Cl}$ \\
\hline Heard about schistosomiasis & $126(88.7)$ & 105(97.2) & 4.4 & $1.3,9.6^{*}$ & $140(89.2)$ & $91(97.8)$ & 5.5 & $1.2,24.5^{*}$ \\
\hline \multicolumn{9}{|l|}{ Signs and symptoms: } \\
\hline At least one symptom & $86(60.6)$ & $71(66.4)$ & 1.3 & $0.8,2.2$ & $97(61.8)$ & $60(64.5)$ & 1.1 & $0.7,1.9$ \\
\hline Haematuria & $41(28.7)$ & $51(47.7)$ & 2.3 & $1.3,3.8^{*}$ & 49(31.0) & $43(46.7)$ & 2.0 & $1.1,3.3^{*}$ \\
\hline Abdominal pain & $58(40.8)$ & $31(29.0)$ & 0.6 & $0.3,1.0$ & $62(39.5)$ & $28(30.1)$ & 0.7 & $0.4,1.1$ \\
\hline Burning urination & 23(16.1) & 19(17.8) & 1.1 & $0.6,2.2$ & 25(15.9) & $17(18.3)$ & 1.2 & $0.6,2.3$ \\
\hline Blood in stool & 26(18.3) & 15(14.0) & 0.7 & $0.4,1.5$ & 25(15.9) & 16(17.2) & 1.1 & $0.6,2.2$ \\
\hline Itching & 18(12.7) & 15(14.0) & 1.1 & $0.5,2.3$ & 19(12.1) & 14(15.2) & 1.3 & $0.6,2.7$ \\
\hline Pale face (anaemia) & 16(11.3) & 17(15.6) & 1.5 & $0.7,3.1$ & $20(12.7)$ & 13(14.0) & 1.1 & $0.5,2.4$ \\
\hline Fatigue & $12(14.8)$ & 1099.3) & 0.6 & $0.3,1.3$ & 25(15.9) & $6(6.5)$ & 0.4 & $0.1,0.9^{*}$ \\
\hline Swollen abdomen & $13(9.2)$ & $12(11.2)$ & 1.3 & $0.5,2.8$ & 14(8.9) & 11(11.8) & 1.4 & $0.6,3.2$ \\
\hline Diarrhoea & $39(27.5)$ & $44(40.7)$ & 1.8 & $1.1,3.1^{*}$ & $43(27.4)$ & $40(43.0)$ & 2.0 & $1.2,3.4^{*}$ \\
\hline Loss of appetite & $12(8.4)$ & 21(19.4) & 2.6 & $1.2,5.6^{*}$ & $15(9.5)$ & 18(19.4) & 2.3 & $1.1,4.8^{*}$ \\
\hline Fever & $36(25.2)$ & $28(26.2)$ & 1.1 & $0.6,1.9$ & $43(27.4)$ & $21(22.6)$ & 0.8 & $0.4,1.4$ \\
\hline Vomiting & 16(11.3) & 15(13.9) & 1.3 & $0.6,2.7$ & 17(10.8) & 14(15.2) & 1.5 & $0.7,3.2$ \\
\hline Dysentery & $1(0.7)$ & $15(14)$ & 13.0 & $3.0,37.0^{*}$ & $6(3.8)$ & 11(11.8) & 3.4 & $1.2,9.5^{*}$ \\
\hline \multicolumn{9}{|l|}{ Transmission: } \\
\hline At least one way of transmission & $60(42.0)$ & $54(50.0)$ & 1.4 & $0.8,2.3$ & $69(43.7)$ & $45(48.9)$ & 1.2 & $0.7,2.1$ \\
\hline Swimming / bathing / playing in infested water & $32(22.5)$ & $34(31.8)$ & 1.6 & $0.9,2.8$ & $38(24.1)$ & $29(31.2)$ & 1.4 & $0.8,2.5$ \\
\hline Snail & 14(9.9) & 11(10.3) & 1.0 & $0.5,2.4$ & 18(11.5) & $8(8.6)$ & 0.7 & $0.3,1.7$ \\
\hline Playing with soil & $34(23.9)$ & 43(39.8) & 2.1 & $1.2,3.6^{*}$ & $40(25.5)$ & 37(39.8) & 1.9 & $1.1,3.3^{*}$ \\
\hline Dirty hands & 28(19.6) & 29(26.9) & 1.5 & $0.8,2.7$ & $35(22.2)$ & $22(23.7)$ & 1.1 & $0.6,2.0$ \\
\hline Eating contaminated food & $34(23.8)$ & 23(21.3) & 0.9 & $0.5,1.6$ & $39(24.8)$ & 18(19.4) & 0.7 & $0.4,1.4$ \\
\hline Drinking untreated water & 14(9.9) & 19(17.8) & 1.9 & $0.9,4.1$ & 22(13.9) & 12(12.9) & 0.9 & $0.4,1.9$ \\
\hline \multicolumn{9}{|l|}{ Prevention: } \\
\hline At least one preventive measure & $62(43.7)$ & $51(47.2)$ & 1.2 & $0.7,1.9$ & $67(42.7)$ & $46(49.5)$ & 1.3 & $0.8,2.2$ \\
\hline Avoid swimming/playing in ponds/streams & 28(19.7) & 36(33.6) & 2.1 & $1.2,3.7^{*}$ & $37(23.6)$ & $27(29.3)$ & 1.3 & $0.8,2.4$ \\
\hline Avoid washing clothes in ponds/streams & 15(10.5) & $11(10.2)$ & 1.0 & $0.4,2.2$ & $20(12.7)$ & $5(5.4)$ & 0.4 & $0.1,1.1$ \\
\hline Avoid playing with soil & $33(23.2)$ & $38(35.5)$ & 1.8 & $1.0,3.2^{*}$ & $38(24.2)$ & $34(36.6)$ & 1.8 & $1.0,3.2^{*}$ \\
\hline Washing hands before eating & 28(19.6) & $39(36.1)$ & 2.3 & $1.3,4.1^{*}$ & $35(22.3)$ & $31(33.3)$ & 1.7 & $0.9,3.1$ \\
\hline Avoid drinking untreated water & 15(10.6) & 18(16.7) & 1.7 & $0.8,3.5$ & 16(10.2) & 17(18.3) & 1.9 & $0.9,4.1$ \\
\hline Washing vegetables/fruit before eating & 29(20.4) & $24(22.2)$ & 1.1 & $0.6,2.0$ & $34(21.5)$ & 19(20.7) & 0.9 & $0.5,1.8$ \\
\hline Taking anti-schistosomal drugs & 19(13.4) & 23(21.3) & 1.7 & $0.9,3.4$ & 19(12.1) & $24(25.8)$ & 2.5 & $1.3,4.9^{*}$ \\
\hline
\end{tabular}

All values are number (\%). OR, Odds ratio. $\mathrm{Cl}$, Confidence interval. *Significant association $(P<0.05)$

\section{Multivariate analysis}

The results of multiple logistic regression analyses for the factors significantly associated with the KAP on schistosomiasis among the Yemeni participants showed that the educational level of the respondents was the most important factor as it was significantly associated with many KAP items. The results indicated that respondents who had at least 6 years of formal education had significantly higher odds of hearing about schistosomiasis $(\mathrm{OR}=3.84 ; 95 \% \mathrm{CI}=1.25,11.81)$, had knowledge about the signs and symptoms $(\mathrm{OR}=3.37 ; 95 \%$ $\mathrm{CI}=1.89,6.01$ ), had knowledge about the presence of blood in stools $(\mathrm{OR}=3.66 ; 95 \% \mathrm{CI}=1.60,8.38)$ and diarrhoea $(\mathrm{OR}=2.06$; $95 \% \mathrm{CI}=1.12,3.78)$ as symptoms of schistosomiasis; had knowledge concerning the role of snails in the transmission of schistosomiasis 
Table 6 Associations of attitude and practices of the participants towards schistosomiasis with their age and educational level

\begin{tabular}{|c|c|c|c|c|c|c|c|c|}
\hline \multirow[t]{2}{*}{ Variable } & \multicolumn{4}{|c|}{ Age (years) } & \multicolumn{4}{|c|}{ Educational level } \\
\hline & $>=40$ & $<40$ & OR & $95 \% \mathrm{Cl}$ & Non educated & Educated & OR & $95 \% \mathrm{Cl}$ \\
\hline \multicolumn{9}{|l|}{ Attitude: } \\
\hline Serious disease & 108(82.4) & $71(70.3)$ & 0.5 & $0.3,0.9^{*}$ & $71(74.7)$ & 107(79.3) & 1.2 & $0.7,2.4$ \\
\hline \multicolumn{9}{|l|}{ Practices: } \\
\hline Swimming/bathing in open water source & $34(24.8)$ & $33(28.9)$ & 1.2 & $0.7,2.2$ & $24(22.0)$ & $42(29.8)$ & 1.5 & $0.8,2.7$ \\
\hline Indiscriminate defaecation/urination & $72(52.9)$ & $76(66.7)$ & 1.8 & $1.1,3.0^{*}$ & $86(78.2)$ & $62(44.3)$ & 0.2 & $0.1,0.4^{*}$ \\
\hline Fetching water from ponds/streams & $70(51.1)$ & $69(61.1)$ & 1.5 & $0.9,2.5$ & $58(53.2)$ & $81(57.4)$ & 1.2 & $0.7,2.0$ \\
\hline Washing clothes \& utensil in open water & $36(26.3)$ & $47(41.6)$ & 2.0 & $1.2,3.4^{*}$ & $35(32.1)$ & $48(34.0)$ & 1.1 & $0.6,1.9$ \\
\hline Wearing shoes when go outside & $100(73.5)$ & $85(75.2)$ & 1.1 & $0.6,1.9$ & $80(73.4)$ & $106(75.2)$ & 1.1 & $0.6,1.9$ \\
\hline Drinking untreated water & 96(70.6) & $77(67.5)$ & 0.9 & $0.5,1.5$ & $83(75.5)$ & $90(63.8)$ & 0.6 & $0.3,0.9^{*}$ \\
\hline Seeking treatment from clinic for GIT and urinary tract symptoms & 109(79.6) & $94(83.2)$ & 1.3 & $0.7,2.4$ & $84(76.4)$ & $119(85.0)$ & 1.8 & $0.9,3.3$ \\
\hline
\end{tabular}

All values are number (\%). OR, Odds ratio. $\mathrm{Cl}$, Confidence interval. *Significant association $(P<0.05)$

$(\mathrm{OR}=3.91 ; 95 \% \mathrm{CI}=1.41,10.91)$; had knowledge about the prevention of schistosomiasis $(\mathrm{OR}=1.85$; $95 \% \mathrm{CI}=1.04,3.28)$; and had knowledge about taking anti-schistosomal drugs as a preventive measure $(\mathrm{OR}=3.17 ; 95 \% \mathrm{CI}=1.36,7.39)$ when compared with non-educated respondents.

Logistic regression analyses also showed that respondents who had a history of schistosomiasis showed higher odds of having knowledge about a swollen abdomen $(\mathrm{OR}=4.39 ; 95 \% \mathrm{CI}=1.50,12.84)$ and anaemia $(\mathrm{OR}=2.73$; $95 \% \mathrm{CI}=1.10,6.75)$ as symptoms; swimming or playing in open water sources as a mode of transmission $(\mathrm{OR}=2.77$; $95 \% \mathrm{CI}=1.24,6.17)$; and had knowledge about the prevention of schistosomiasis $(\mathrm{OR}=2.42 ; 95 \% \mathrm{CI}=1.31$, 4.47) when compared with those with no previous history of infection. Moreover, respondents aged below 40 years were less likely to hear about schistosomiasis $(\mathrm{OR}=0.31 ; 95 \%$ $\mathrm{CI}=0.10,0.78)$.

\section{Discussion}

The present study is the first to provide information about the knowledge, attitude and practices (KAP) concerning schistosomiasis in Yemen. Despite the intensive efforts to control the disease, schistosomiasis is still highly prevalent among children in rural Yemen and our previously published work revealed that $31.8 \%$ of the participants were infected with Schistosoma species [10]. It was found that the overall prevalence was $22.5 \%$ for S. haematobium, $8.0 \%$ for S. mansoni, and $1.3 \%$ for coinfection with both S. haematobium and S. mansoni. Although the majority of the respondents had heard about schistosomiasis, the results showed that awareness about the symptoms, ways of transmission and preventive measures among the participants was generally poor. The present study was carried out in endemic areas that are undergoing active control and prevention surveillance by the schistosomiasis national control project (SNCP), which

Table 7 Associations of attitude and practices of the participants towards schistosomiasis with their employment status and household monthly income

\begin{tabular}{|c|c|c|c|c|c|c|c|c|}
\hline \multirow[t]{2}{*}{ Variable } & \multicolumn{4}{|c|}{ Employment status } & \multicolumn{4}{|c|}{ Household monthly income } \\
\hline & Not working & working & OR & $95 \% \mathrm{Cl}$ & $<Y R 20,000$ & $\geq Y R 20,000$ & OR & $95 \% \mathrm{Cl}$ \\
\hline \multicolumn{9}{|l|}{ Attitude: } \\
\hline Serious disease & $92(73.0)$ & $86(82.7)$ & 1.8 & $0.9,3.4$ & $105(75.0)$ & $73(80.2)$ & 1.4 & $0.7,2.6$ \\
\hline \multicolumn{9}{|l|}{ Practices: } \\
\hline Swimming/bathing in open water source & $32(22.5)$ & $34(31.8)$ & 1.6 & $0.9,2.8$ & $38(24.1)$ & 29(31.2) & 1.4 & $0.8,2.5$ \\
\hline Indiscriminate defaecation/urination & $108(75.5)$ & $40(37.4)$ & 0.2 & $0.2,0.3^{*}$ & $120(76.4)$ & $28(30.1)$ & 0.1 & $0.1,0.2^{*}$ \\
\hline Fetching water from ponds/streams & $78(54.4)$ & $61(57.0)$ & 1.1 & $0.7,1.8$ & $88(55.7)$ & $51(54.8)$ & 1.0 & $0.6,1.6$ \\
\hline Washing clothes \& utensil in open water & $41(28.7)$ & $43(39.8)$ & 1.6 & $0.9,2.8$ & $47(29.7)$ & $36(39.1)$ & 1.5 & $0.9,2.6$ \\
\hline Wearing shoes when outside & $81(57.0)$ & $105(97.2)$ & 19.4 & $8.0,47.1^{*}$ & $100(63.7)$ & $86(92.5)$ & 7.0 & $3.0,16.2^{*}$ \\
\hline Drinking untreated water & $100(70.4)$ & 73(67.6) & 0.9 & $0.5,1.5$ & $108(68.8)$ & $65(69.9)$ & 1.1 & $0.6,1.8$ \\
\hline $\begin{array}{l}\text { Seeking treatment from clinic for GIT and urinary } \\
\text { tract symptoms }\end{array}$ & $110(77.5)$ & $93(86.1)$ & 1.8 & $0.9,2.5$ & $124(79.0)$ & $79(84.9)$ & 1.5 & $0.8,3.0$ \\
\hline
\end{tabular}

All values are number (\%). OR, Odds ratio. $\mathrm{Cl}$, Confidence interval. *Significant association $(P<0.05)$ 
may explain why $92.4 \%$ of the respondents had heard about the disease. This is also consistent with the finding that half of the respondents declared a history of infection among their children, which supports the endemicity of infection in these communities. This was also supported by the significantly higher level of schistosomiasis-related knowledge among respondents who had infected children. Our findings showed that two-thirds of the respondents indicated that they had heard about the disease from the control programme personnel while $21.7 \%$ cited the clinics and hospitals as the source of their information. Moreover, the present study revealed poor contribution from the media and schools in Yemen, which should receive proper attention as involvement of the media and schools is imperative in the battle against this devastating disease.

Overall, these findings are in agreement with previous studies from other schistosomiasis-endemic countries; a high level of awareness of schistosomiasis has been reported in Brazil [22], Egypt [23] and Kenya [24]. In contrast, poor awareness about schistosomiasis has been reported in Malawi [25], Zimbabwe [15] and Western Kenya [26]. Despite seven years of health education interventions using a diversity of communication outlets including radio, television and posters, a previous study in Senegal revealed that although $86 \%$ of the respondents stated that they had heard about schistosomiasis, only $30 \%$ had adequate knowledge about the symptoms and modes of transmission of the disease [27].

The present study revealed poor knowledge about the modes of transmission and preventive measures of schistosomiasis; particularly the role of snails in the transmission of schistosomiasis. When the snails were shown to the respondents, most of them declared that there are plenty of these creatures in the streams, ponds and irrigation canals and also mentioned that "snails are not harmful and usually our children like to collect and play with them". In western Kenya, a previous study found that some of the participants knew that snails and poor sanitation contributed to the spread of the disease, but lacked understanding of the transmission cycle [26]. Hence, it is clear that the lack of this knowledge among the targeted population may create an additional burden and cost for controlling the disease and may cause the failure of the schistosomiasis eradication programme.

Our findings revealed that one-third of the respondents could not associate the infection with any symptom. Conversely, previous studies from Brazil and Ethiopia reported diverging information where the majority of the subjects were able to associate these symptoms with the infection $[22,28]$. Similarly, it is also worth noting that knowledge about the symptoms of intestinal schistosomiasis among the respondents was negligible, as only $17.7 \%$ of them mentioned blood in stools. This could be attributed to the disease being frequently confused with other intestinal infections exhibiting similar symptoms, such as amoebic dysentery, which is common among the targeted populations $[29,30]$. Another reason could be the lower prevalence of intestinal schistosomiasis compared to urogenital schistosomiasis.

In respect of treatment-seeking behaviour, the majority of the participants seek treatment for haematuria and GIT symptoms at health centres. However, time is a major concern as all such participants acknowledged that there is always a delay in seeking treatment because many people do not seek care for symptoms until they become severe. Poverty contributes significantly to this delay, as the patients may not earn enough to pay for the cost of transport and medical services. In addition, infected individuals may only decide to seek treatment from health centres when they cannot tolerate the symptoms of the disease any longer and they receive treatment at a late stage of the disease. This is especially detrimental considering that morbidity in schistosomiasis is a function of the infection intensity and duration. In addition, this could also be attributed to the poor knowledge about the fatal complications of schistosomiasis [24]. Furthermore, the present study showed that $14.3 \%$ of the respondents thought that treating schistosomiasis at a health centre is very expensive and cited this as a reason for seeking alternative treatment. Similar findings and cited reasons were reported in Western Kenya and Uganda [24, 31]. Previous studies in Ghana showed that more than $70 \%$ of the schistosomiasis-infected individuals opt for traditional self-treatment without visiting a health facility, with 'Do not have the money' and 'Not serious enough' being the commonest reasons for not visiting a clinic $[32,33]$.

It should be noted that a considerable number of participants in these communities showed confusion between the schistosomiasis and soil-transmitted helminth (STH) infections, which are also endemic in these areas. This could be attributed to the practice of the MDA that was carried out by the SNCP 2010-2016, which covered both diseases. However, this may lead to serious complications in patients who believe that schistosomiasis can be cured by taking albendazole and could contribute to the transmission of the disease within endemic communities. Similarly, some of the respondents questioned the usefulness of the drug as it cannot prevent them from reinfection. For this reason, 11 respondents (four males and seven females, aged $>50$ years) declared that they were suffering with haematuria but do nothing about its treatment.

Our study also reported many mistaken beliefs about schistosomiasis as a large number of the respondents demonstrated misconceptions about the transmission and prevention of schistosomiasis. For instance, a noteworthy number of participants believed that schistosomiasis is 
transmitted by playing with soil, eating contaminated food and from dirty hands. Likewise, similar percentages of the respondents mentioned avoiding playing with soil, washing hands before eating and washing vegetables/fruits before consumption for the prevention of schistosomiasis. Since 2006, Praziquantel has been distributed together with albendazole tablets to protect against STH infections, which are highly endemic in these areas [34]. When questioned about the history of deworming, many of the respondents described the two orange flavoured tablets (albendazole).

Interestingly, our findings showed that less than half of the participants believed that schistosomiasis is a preventable disease, while one third of them believed that acquiring schistosomiasis could not be prevented. Besides poor health education, this could be due to continuous exposure and reinfection after repeated deworming in these communities. Furthermore, our findings showed that only $13 \%$ of the respondents knew that faeces and urine are the sources of infection while three-quarters did not know. This indicates a lack of health education about the causes and prevention of schistosomiasis among these people, which should be provided during the mass drug administration campaigns.

In investigating the factors associated with the respondents' KAP, we found that educated, working and those who had $\geq$ YR20, 000 monthly income had significantly better knowledge about the signs and symptoms, transmission (snail) and prevention of schistosomiasis when compared to their counterparts. Education plays an important role in people's perceptions and practices of controlling schistosomiasis [35]. Previous studies from Africa and Asia showed that the odds of having a lower knowledge about schistosomiasis were significantly higher in the respondents who had a primary education level or below [36, 37]. In agreement with these findings, our study showed a significant impact for the level of education on the population's KAP concerning schistosomiasis. This could be attributed to the ability of reading and understanding the leaflets and posters distributed by the control programme. In contrast, previous studies from Cote d'Ivoire and Uganda found no significant association between the educational level and the level of knowledge of schistosomiasis [16, 31]. Our results showed that only four respondents declared schools as the source of information about schistosomiasis, which needs attention, as the school can play an important role in controlling different diseases among the targeted communities. This can be done through health promoting programmes that teach the students how to protect themselves against infections and then disseminate information to the surrounding communities $[38,39]$.

In respect of gender, our findings revealed a similar level of knowledge and attitude towards schistosomiasis between the male and female respondents. This is in accordance with our previous findings that there was no significant difference in the prevalence of schistosomiasis among males and females [10]. However, previous studies from Malawi, Zanzibar and South Darfur reported a significantly higher prevalence of infection and better knowledge about the disease among male participants compared to females [40-42]. This could be due to some religious and cultural beliefs. In Islamic communities, females are not allowed to swim or bathe in the open water sources and are not allowed to participate in fishing and irrigation activities [43, 44]. However, in rural Yemen, females are responsible for fetching water and washing clothes and utensils at streams, ponds, dams and other water sources, as was observed in the study area [10]. It is well known that poverty propagates the occurrence of many health problems including NTDs in developing communities. Within this context, our findings revealed a significantly higher level of knowledge and attitude towards schistosomiasis among respondents who had a household monthly income of $\geq$ YR20, 000 compared to those with a low income.

Recently, the WHO called for a global effort to eliminate human schistosomiasis by 2025, with MDA as a cardinal intervention [45]. However, this call has underscored the need for more emphasis on snail-related research [46]. Despite the potential threat of drug resistance developing in the schistosomes [47], MDA has been used for generations as the main pillar and the most cost-effective intervention to control schistosomiasis; however, chemotherapy alone may never achieve transmission control or elimination [48, 49]. Thus, other interventions, such as snail control and health education among schistosomiasisendemic communities are essential and should be implemented in parallel with MDA [50, 51]. Adema et al. [46] raised some issues pertinent to snail control, such as a decline in snail related funding and a concomitant decline, to a shockingly low level, as well as the availability of investigators and young workers who are able to identify medically relevant snails. Great success has been achieved in many countries, such as China and Egypt, in reducing the transmission, prevalence and morbidity of schistosomiasis $[36,52,53]$. The MDA-based strategy was fundamental in this success hand-in-hand with health education campaigns and capacity building through the training of personnel working in rural health units or involved in reducing the roles of humans and animals as sources of infection for snails $[52,53]$. Similar success can also be achieved in Yemen through an integrated national control approach that should consider the MDA, snail control and health education. Rural communities in Yemen share similar socioeconomic and health profiles with a different climate. Coastal plains and foothills (Taiz, Ibb and Hodeidah) have more streams, whereas mountainous areas (Sana'a and Dhamar) have more 
water pools/troughs and dams. Thus, we believe that our findings are generalisable to the entire rural population in Yemen. However, further studies in other provinces are required to confirm this conjecture.

\section{Conclusion}

This study reveals inadequate knowledge, attitude and practices concerning schistosomiasis among the rural population residing in schistosomiasis endemic areas in Yemen, which could be a challenging obstacle to the endeavour towards the elimination of schistosomiasis from Yemen. The study also shows an alarmingly high prevalence of schistosomiasis among children in these areas. Thus, there is a great need for a proper health education intervention and community mobilisation in order to enhance prevention and instil better knowledge concerning the transmission and prevention of schistosomiasis. Providing efficient health education to people residing in schistosomiasis endemic areas is imperative for an effective and sustainable control programme in order to save the lives and future of the most vulnerable children in rural Yemen.

\section{Competing interests}

The authors have declared that no competing interests exist.

\section{Authors' contributions}

HMA, KHC and JS designed and supervised the study. HS conducted the sampling and field survey. HMA and HS analysed the data and wrote the paper. WMA, AKA, NAN, SD, MAA, and AMA were involved in the collection and laboratory examination of samples. KHC, JS and II revised the manuscript. All authors approved the final version of the manuscript.

\section{Acknowledgements}

We gratefully acknowledge the Schistosomiasis National Control Project offices (Taiz, Ibb, Dhamar, Sana'a and Hodeidah), Ministry of Health and Population, Yemen for their generous cooperation during this study. We also wish to express our appreciation to the parents and their children for their voluntary participation in this study. The work presented in this paper was funded by the University of Malaya High Impact Research Grant UM-MOHE UM.C/625/1/HIR/MOHE/MED/18 from the Ministry of Higher Education Malaysia, and also by the University of Malaya Research Grants; RG331-15AFR.

\section{Author details \\ ${ }^{1}$ Department of Parasitology, Faculty of Medicine, University of Malaya, 50603 Kuala Lumpur, Malaysia. ${ }^{2}$ Department of Medical Laboratories, Faculty of Medical Sciences, Hodeidah University, Hodeidah, Yemen. ${ }^{3}$ Azal National Research Center, Azal University for Human Development, 447 Sana'a, Yemen. ${ }^{4}$ Department of Parasitology, Faculty of Medicine and Health Sciences, Sana'a University, 1247 Sana'a, Yemen. ${ }^{5}$ Department of Biomedical Science, Faculty of Medicine, University of Malaya, 50603 Kuala Lumpur, Malaysia.}

Received: 2 July 2015 Accepted: 17 August 2015

Published online: 25 August 2015

\section{References}

1. World Health Organization. Schistosomiasis: number of people treated in 2011. Wkly Epidemiol Rec. 2013:88:81-8.

2. Hotez PJ, Savioli L, Fenwick A. Neglected tropical diseases of the Middle East and North Africa: Review of their prevalence, distribution, and opportunities for control. PLoS Negl Trop Dis. 2012;6(2):e1475. doi:10.1371/journal.pntd.0001475.

3. Mostafa MH, Sheweita SA, O'Connor PJ. Relationship between schistosomiasis and bladder cancer. Clin Microbiol Rev. 1999;12:97-111.
4. van der Werf MJ, de Vlas SJ, Brooker S, Looman CWN, Nagelkerke NJD, Habbema JDF, et al. Quantification of clinical morbidity associated with schistosome infection in sub-Saharan Africa. Acta Trop. 2003;86:125-39.

5. Steinmann P, Keiser J, Bos R, Tanner M, Utzinger J. Schistosomiasis and water resources development: systematic review, meta-analysis, and estimates of people at risk. Lancet Infect Dis. 2006;6:411-25.

6. The World Bank. Projects and operations. Yemen schistosomiasis. http://www.worldbank.org/projects/P113102/yemen-schistosomiasis?lang=en. Accessed 12 Jul 2015

7. Ministry of Public Health and Population, Yemen. Schistosomiasis national project. http://www.mophp-ye.org/arabic/docs/Yemen_SCP_PAD_Arabic.pdf. Accessed 5 Jun 2015.

8. Baring E, Hotez PJ. Yemen: Fighting neglected tropical diseases against all odds. PLoS Negl Trop Dis. 2014;8(12):e3292. doi:10.1371/journal.pntd.0003292.

9. World Health Organization. Working to overcome the global impact of neglected tropical diseases: first WHO report on neglected tropical diseases. Geneva: WHO; 2010

10. Sady H, Al-Mekhlafi HM, Mahdy MA, Lim YA, Mahmud R, Surin J. Prevalence and associated factors of Schistosomiasis among children in Yemen: implications for an effective control programme. PLoS Negl Trop Dis. 2013;7(8):e2377. doi:10.1371/journal.pntd.0002377.

11. Al-Samawi AS, Aulaqi SM. Urinary Bladder Cancer in Yemen. Oman Med J. 2013;28:337-40. doi:10.5001/omj.2013.97.

12. Govere J, Durrheim D, Grange Kl, Mabuza A, Booman M. Community knowledge and perceptions about malaria and practices influencing malaria control in Mpumalanga province, South Africa. S Afr Med J. 2000:90:611-16.

13. Joshi $A B$, Banjara MR. Malaria related knowledge, practices and behaviour of people in Nepal. J Vector Borne Dis. 2008;45:44-50.

14. Nasr NA, Al-Mekhlafi HM, Ahmed A, Roslan MA, Bulgiba A. Towards an effective control programme of soil-transmitted helminth infections among Orang Asli in rural Malaysia. Part 2: Knowledge, attitude, and practices. Parasit Vectors. 2013;6:28. doi:10.1186/1756-3305-6-28.

15. Midzi N, Mtapuri-Zinyowera S, Mapingure MP, Paul NH, Sangweme D, Hlerema $G$, et al. Knowledge, attitudes and practices of grade three primary schoolchildren in relation to schistosomiasis, soil-transmitted helminthiasis and malaria in Zimbabwe. BMC Infect Dis. 2011;11:169. doi:10.1186/14712334-11-169.

16. Acka CA, Raso G, N'Goran EK, Tschannen AB, Bogoch II, Seraphin E, et al. Parasitic worms: knowledge, attitudes, and practices in western Cote d'Ivoire with implications for integrated control. PLoS Negl Trop Dis. 2010;4:e910. doi:10.1371/journal.pntd.0000910.

17. World Bank. World Development Indicators 2010. Washington, DC: World Bank; 2010.

18. Cheesbrough M. District laboratory practice in tropical countries, part 1. 2nd ed. Cambridge: Cambridge University Press; 2009. p. 196-220.

19. Kosinski KC, Bosompem KM, Stadecker MJ, Wagner AD, Plummer J, Durant JL, et al. Diagnostic accuracy of urine filtration and dipstick tests for Schistosoma haematobium infection in a lightly infected population of Ghanaian schoolchildren. Acta Trop. 2011;118:123-27. doi:10.1016/ j.actatropica.2011.02.006

20. World Health Organization. Helminth control in school-age children: A guide for managers of control programmes. Geneva: WHO; 2002.

21. Montresor A. Arithmetic or geometric means of eggs per gram are not appropriate indicators to estimate the impact of control measures in helminth infections. Trans R Soc Trop Med Hyg. 2007;101:773-6.

22. Uchoa E, Barreto SM, Firmo JO, Guerra HL, Pimenta Jr FG, Costa MF L e. The control of schistosomiasis in Brazil: an ethno-epidemiological study of the effectiveness of a community mobilization program for health education. Soc Sci Med. 2000;51:1529-41.

23. Kloos H, Sidrak W, Michael AA, Mohareb EW, Higashi Gl. Disease concepts and treatment practices relating to schistosomiasis hematobium in Upper Egypt. J Trop Med Hyg. 1982;85:99-107.

24. Musuva RM, Awiti A, Omedo M, Ogutu M, Secor WE, Montgomery SP, et al Community knowledge, attitudes and practices on schistosomiasis in western Kenya-the SCORE Project. Am J Trop Med Hyg. 2014;90:646-52. doi:10.4269/ajtmh.13-0488.

25. Poole H, Terlouw DJ, Naunje A, Mzembe K, Stanton M, Betson M, et al. Schistosomiasis in pre-school-age children and their mothers in Chikhwawa district, Malawi with notes on characterization of schistosomes and snails. Parasit Vectors. 2014;7:153. doi:10.1186/1756-3305-7-153. 
26. Odhiambo GO, Musuva RM, Atuncha VO, Mutete ET, Odiere MR, Onyango RO, et al. Low levels of awareness despite high prevalence of schistosomiasis among communities in Nyalenda informal settlement, Kisumu City, Western Kenya. PLoS Negl Trop Dis. 2014;8(4):e2784. doi:10.1371/journal.pntd.0002784

27. Sow S, de Vlas SJ, Mbaye A, Polman K, Gryseels B. Low awareness of intestinal schistosomiasis in northern Senegal after 7 years of health education as part of intense control and research activities. Trop Med Int Health. 2003:8:744-9.

28. Geleta S, Alemu A, Getie S, Mekonnen Z, Erko B. Prevalence of urinary schistosomiasis and associated risk factors among Abobo primary school children in Gambella Regional State, southwestern Ethiopia: a cross sectional study. Parasit Vectors. 2015;8:215. doi:10.1186/s13071-015-0822-5.

29. Azazy AA, Al-Mahbashi TY, Al-Mekhlafi HM. Prevalence of intestinal and blood parasites among school children in Sana'a and Al-Mahweet provinces, Yemen. Yemen Med J. 2002:4:50-5.

30. Alyousefi NA, Mahdy MA, Mahmud R, Lim YA. Factors associated with high prevalence of intestinal protozoan infections among patients in Sana'a City, Yemen. PLoS One. 2011;6(7):e22044. doi:10.1371/journal.pone.0022044.

31. Kabatereine N, Fleming F, Thuo W, Tinkitina B, Tukahebwa EM, Fenwick A. Community perceptions, attitude, practices and treatment seeking behaviour for schistosomiasis in L.Victoria islands in Uganda. BMC Res Notes. 2014;7:900. doi:10.1186/1756-0500-7-900.

32. Danso-Appiah A, De Vlas SJ, Bosompem KM, Habbema JD. Determinants of health-seeking behaviour for schistosomiasis-related symptoms in the context of integrating schistosomiasis control within the regular health services in Ghana. Trop Med Int Health. 2004;9(7):784-94.

33. Danso-Appiah A, Stolk WA, Bosompem KM, Joseph Otchere J, Looman CW, Habbema JD, et al. Health seeking behaviour and utilization of health facilities for schistosomiasis related symptoms in Ghana. PLoS Negl Trop Dis. 2010:4:e867. doi:10.1371/journal.pntd.0000867.

34. Oshish A, AlKohlani A, Hamed A, Kamel N, AlSoofi A, Farouk H, et al. Towards nationwide control of schistosomiasis in Yemen: a pilot project to expand treatment to the whole community. Trans R Soc Trop Med Hyg. 2011;105:617-27. doi:10.1016/j.trstmh.2011.07.013.

35. Adoka SO, Anyona DN, Abuom PO, Dida GO, Karanja D, Vulule JM, et al. Community perceptions of schistosomiasis transmission, prevalence and control in relation to aquatic habitats in the Lake Victoria basin of Kenya. East Afr Med J. 2014;91:232-44.

36. Liu L, Yang GJ, Zhu HR, Yang K, Ai L. Knowledge of, attitudes towards, and practice relating to schistosomiasis in two subtypes of a mountainous region of the People's Republic of China. Infect Dis Poverty. 2014;3:16. doi:10.1186/2049-9957-3-16.

37. Yirenya-Tawiah DR, Annang T, Otchere J, Bentum D, Edoh D, Amoah C, et al. Urinary schistosomiasis among adults in the Volta Basin of Ghana: prevalence, knowledge and practices. J Trop Med Parasitol. 2011;34(1):1-16.

38. Yuan LP, Manderson L, Ren MY, Li GP, Yu DB, Fang JC. School-based interventions to enhance knowledge and improve case management of schistosomiasis: a case study from Hunan, China. Acta Trop. 2005;96(2-3):248-54.

39. Gyorkos TW, Maheu-Giroux M, Blouin B, Casapia M. Impact of health education on soil-transmitted helminth infections in schoolchildren of the Peruvian Amazon: a cluster-randomized controlled trial. PLoS Negl Trop Dis. 2013;7(9):e2397. doi:10.1371/journal.pntd.0002397.

40. Kapito-Tembo AP, Mwapasa V, Meshnick SR, Samanyika Y, Banda D, Bowie $C$, et al. Prevalence distribution and risk factors for Schistosoma hematobium infection among school children in Blantyre, Malawi. PLoS Negl Trop Dis. 2009;3(1):e361. doi:10.1371/journal.pntd.0000361.

41. Rudge JW, Stothard JR, Basanez MG, Mgeni AF, Khamis IS, Khamis AN, et al. Micro-epidemiology of urinary schistosomiasis in Zanzibar: Local risk factors associated with distribution of infections among schoolchildren and relevance for control. Acta Trop. 2008;105:45-4

42. Deribe K, Eldaw A, Hadziabduli S, Kailie E, Omer MD, Mohammed AE, et al. High prevalence of urinary schistosomiasis in two communities in South Darfur: implication for interventions. Parasit Vectors. 2011;4:14. doi:10.1186/ 1756-3305-4-14.

43. Evans DS, King JD, Eigege A, Umaru J, Adamani W, Alphonsus K, et al. Assessing the $\mathrm{WHO} 50 \%$ prevalence threshold in school-aged children as indication for treatment of urogenital schistosomiasis in Adults in central Nigeria. Am J Trop Med Hyg. 2013;88(3):441-5. doi:10.4269/ajtmh.12-0511.

44. Senghor B, Diallo A, Sylla SN, Doucouré S, Ndiath MO, Gaayeb L, et al. Prevalence and intensity of urinary schistosomiasis among school children in the district of Niakhar, region of Fatick, Senegal. Parasit Vectors. 2014;7:5 doi:10.1186/1756-3305-7-5

45. World Health Organization. Accelerating work to overcome the global impact of neglected tropical diseases-a roadmap for implementation. Geneva: WHO; 2012. http://www.who.int/entity/neglected_diseases/ NTD_RoadMap_2012_Fullversion.pdf. Accessed 20 Jun 2015.

46. Adema CM, Bayne CJ, Bridger JM, Knight M, Loker ES, Yoshino TP, et al. Wil all scientists working on snails and the diseases they transmit please stand up? PLoS Negl Trop Dis. 2012;6(12):e1835. doi:10.1371/journal.pntd.0001835.

47. Seto EY, Wong BK, Lu D, Zhong B. Human schistosomiasis resistance to praziquantel in China: should we be worried? Am J Trop Med Hyg. 2011:85:74-82. doi:10.4269/ajtmh.2011.10-0542

48. Gray DJ, Mcanus DP, Li Y, Williams GM, Bergquist R, Ross AG. Schistosomiasis elimination: lessons from the past guide the future. Lancet Infect Dis. 2010;10:733-6. doi:10.1016/S1473-3099(10)70099-2.

49. Ross AG, Olveda RM, Chy D, Olveda DU, Li Y, Harn DA, et al. Can mass drug administration lead to the sustainable control of schistosomiasis? J Infect Dis. 2015;211(2):283-9. doi:10.1093/infdis/jiu416.

50. King CH, Bertsch D. Historical Perspective: Snail Control to Prevent Schistosomiasis. PLoS Negl Trop Dis. 2015;9(4):e0003657. doi:10.1371/ journal.pntd.0003657.

51. Kariuki HC, Madsen $H$, Ouma JH, Butterworth AE, Dunne DW, Booth $M$, et al. Long term study on the effect of mollusciciding with niclosamide in stream habitats on the transmission of schistosomiasis mansoni after communitybased chemotherapy in Makueni District, Kenya. Parasit Vectors. 2013;6:107. doi:10.1186/1756-3305-6-107.

52. Wang LD, Chen HG, Guo JG, Zeng XJ, Hong XL, Xiong JJ, et al. A strategy to control transmission of Schistosoma japonicum in China. N Engl J Med. 2009;360(2):121-8. doi:10.1056/NEJMoa0800135.

53. Barakat RM. Epidemiology of schistosomiasis in Egypt: Travel through time: Review. J Adv Res. 2013;4(5):425-32. doi:10.1016/j.jare.2012.07.003.

\section{Submit your next manuscript to BioMed Central and take full advantage of:}

- Convenient online submission

- Thorough peer review

- No space constraints or color figure charges

- Immediate publication on acceptance

- Inclusion in PubMed, CAS, Scopus and Google Scholar

- Research which is freely available for redistribution 